\title{
Euler-Euler Large Eddy Simulation of a Square Cross-Sectional Bubble Column Using the Neptune_CFD Code
}

\author{
B. Ničeno, ${ }^{1}$ M. Boucker, ${ }^{2}$ and B. L. Smith ${ }^{1}$ \\ ${ }^{1}$ Thermal-Hydraulics Laboratory, Nuclear Energy and Safety Department, Paul Scherrer Institut, 5232 Villigen PSI, Switzerland \\ ${ }^{2}$ Research \& Development/MFEE, Electricité de France(EDF), 6 quai Watier, 78400 Chatou, France
}

Correspondence should be addressed to B. Ničeno, bojan.niceno@psi.ch

Received 5 December 2007; Accepted 28 January 2008

Recommended by Iztok Tiselj

In this work, we report on Euler-Euler large eddy simulation (EELES) of dispersed bubbly flow in a square cross-sectional bubble column. Simulations are performed using the Neptune_CFD package, and results processed using the SALOME platform. The motivation to undertake this study is to check our implementation of the Smagorinsky subgrid-scale (SGS) model into Neptune_CFD. We outline all the physical models used, and we present instantaneous realizations of velocity and void fraction fields in order to illustrate the structure of the turbulence field, and long-time averaged results, to compare with analogous simulations performed using the CFX-4 code and experimental data. The same physical models and constants have been used in both the CFX-4 and Neptune_CFD codes, except the SGS model, which is Smagorinsky in case of Neptune_CFD and a oneequation model in CFX-4. The results obtained with EELES compare reasonably well with experiment, meaning in particular that the implementations have been successful. Some perspectives on the further use of EELES are also given.

Copyright (C) 2009 B. Ničeno et al. This is an open access article distributed under the Creative Commons Attribution License, which permits unrestricted use, distribution, and reproduction in any medium, provided the original work is properly cited.

\section{Introduction}

The aim of the NURESIM [1] integrated project is to provide the initial step towards a common European software platform for analysing, modelling, and exchanging data for nuclear reactor simulations. One of the main goals of the project is the integration of state-of-the-art physical models in a common, open-software platform, including the latest advances in reactor core physics, thermal-hydraulics, and coupled multiphysics modelling capabilities. The objective of the thermal-hydraulic subproject within NURESIM is the improvement of the predictive capabilities of the simulation tools for key two-phase-flow, thermal-hydraulic processes that can occur in nuclear reactors, focusing on two highpriority issues: critical heat flux (CHF) [2] and pressurized thermal shock (PTS) [3]. As a part of this activity, the Paul Scherrer Institute is investigating the potential of the EELES approach for simulating bubbly flows, which are important for understanding CHF and PTS, using the Neptune_CFD code [4], the CFD component of the NURESIM platform. In view of this, it was thought desirable to carry out EELES in a three-dimensional, square-cross-sectional bubble column, based on the experiments of Deen et al. [5], to validate the implementation of the SGS model in the code. In the present work, for reasons of simplicity, the performance of Neptune_CFD with the EELES paradigm is assessed based on the Smagorinsky SGS model. These results, and those obtained with the CFX-4 code, are compared against experimental findings [5]. We point to the potential weaknesses of the current approach, and propose directions for future research and development in the area.

Previous work on EELES (often referred to as two-fluid LES) for bubbly flows include Milelli et al. [6], who used Smagorinsky and dynamic SGS models to study the motion of a bubble plume in a cylindrical test section $50 \mathrm{~cm}$ in diameter, $40 \mathrm{~cm}$ high, for a maximum superficial gas velocity of $6.11 \mathrm{~mm} / \mathrm{s}$. A similar approach was taken by Deen et al. [7] to simulate a bubble plume in a test section with a square cross-section of $15 \mathrm{~cm} \times 15 \mathrm{~cm}$, a height of $45 \mathrm{~cm}$, and superficial gas velocity of $4.9 \mathrm{~mm} / \mathrm{s}$. The authors compare the EELES prediction with those obtained using a $K-\varepsilon$ model and conclude that EELES results are closer to experimental measurements. Turbulent bubbly shear flows have also been analyzed [8] for a square channel $30 \mathrm{~cm}$ wide, $4 \mathrm{~cm}$ deep, 


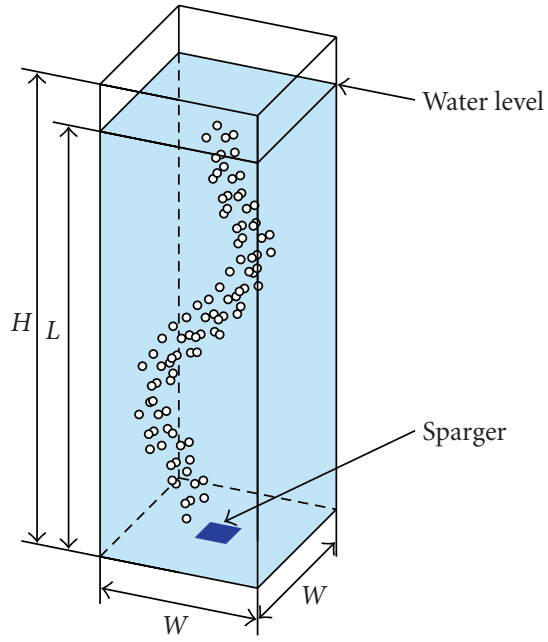

FIgURE 1: Geometry of the Deen bubble column experiment.

and $60 \mathrm{~cm}$ high. A splitter plane was used to divide the inlet channel in two equal parts. A mixture of water and air, with different velocities and void fractions, was introduced at the inlet sections. More recently, an LES calculation incorporating the Smagorinsky SGS model has been reported for a rectangular column [9]. LES predictions were compared with transient results obtained using a mixing length model and the $K-\varepsilon$ model; all three models produced similar results.

1.1. The Deen Bubble Column Experiment. In the Deen series of experiments [5], the hydrodynamics of the bubble plume is studied in the same apparatus, but for different inlet conditions. In the test to be investigated here, bubbles are created by injecting air with a superficial gas velocity of $4.9 \mathrm{~mm} / \mathrm{s}$ through a central sparger at the bottom of the vessel of dimension $5 \mathrm{~cm} \times 5 \mathrm{~cm}$. The experiment operates at atmospheric pressure. A sketch of the Deen experiment is given in Figure 1.

\section{Physical Models}

2.1. Euler-Euler Approach. The governing equation for conservation of mass, with no mass exchange between the phases, takes the form:

$$
\frac{\partial}{\partial t}(r \rho)_{\alpha}+\nabla \cdot(r \rho \mathbf{u})_{\alpha}=0,
$$

in which $t$ is time, $r$ is the volume fraction, $\rho$ is the phase density, $\mathbf{u}$ the phase velocity, and $\alpha$ is the phase indicator. Conservation of momentum is governed by the relation:

$$
\frac{\partial(r \rho \mathbf{u})_{\alpha}}{\partial t}+\nabla \cdot(r \rho \mathbf{u u})_{\alpha}=-\nabla \cdot(r \tau)_{\alpha}-r_{\alpha} \nabla p+(r \rho)_{\alpha} \mathbf{g}+\mathbf{M}_{F, \alpha}
$$

where $\tau$ is shear stress, $p$ is pressure, and $\mathbf{g}$ is the gravity vector. The last term on the right-hand side represents the momentum exchange between the phases due to interface forces and requires modelling. The models we have used are given in Section 2.3.

2.2. LES of Turbulence. Turbulence is modelled in the liquid phase of the flow. The first step in the derivation of the governing equations for LES is the decomposition of the velocity field into resolved (grid scale) and unresolved (SGS) parts. For each component, we write

$$
\mathbf{u}_{L}=\tilde{\mathbf{u}}_{L}-\mathbf{u}_{L}^{\prime},
$$

where the resolved velocity on the left side is equal to the difference between the true instantaneous velocity (first term on the right) and the unresolved velocity (second term on the right). The filtering of the nonlinear advection term on the left-hand side of (2) leads to an additional stress-like term, which has to be modelled. Hence, we write

$$
\tau_{L}=-2(\mu \mathbf{S})_{L}+\tau_{\mathrm{SGS}, L}
$$

The first term represents the laminar stress, with the strain rate tensor defined as

$$
\mathbf{S}_{L}=\frac{1}{2}\left[\nabla \mathbf{u}_{L}+\left(\nabla \mathbf{u}_{L}\right)^{T}\right]
$$

and the second term derives from the turbulence effects and needs closure. In this work, we have used two closure models: Smagorinsky model (in Neptune_CFD), and a one-equation model (in CFX-4).

2.3. Smagorinsky Model. To complete (4), we use a formulation [10] for the turbulent stress:

$$
\tau_{\mathrm{SGS}, L}-\frac{1}{3}\left(\tau_{k k} \delta_{i j}\right)_{L}=-2\left(\mu_{t} \mathrm{~S}\right)_{L}
$$

The second term on the left-hand side, the trace of the SGS stress tensor, is implicitly added to the pressure, while the deviatoric part is modelled by the expression on the righthand side, that is, by the Boussinesq eddy viscosity $\left(\mu_{t}\right)$ concept. The Smagorinsky model is an algebraic one, with the eddy viscosity calculated from:

$$
\mu_{t, L}=\rho_{L}\left(C_{S} \Delta\right)^{2}\left|\mathbf{S}_{L}\right| .
$$

In (7), $\Delta$ is a filter width, associated with the cell size. In the present work, it was estimated as the cube root of the cell volume:

$$
\Delta=V^{1 / 3}
$$

although other definitions are possible. $C_{S}$ is the Smagorinsky constant, and was set here to 0.12 since the flow is dominated by the large-scale structures generated by the buoyancy force, as pointed out in [11]. The eddy viscosity, defined by (7), is used in the stress tensor appearing in the momentum (4), giving

$$
\tau_{L}=-2\left[\left(\mu+\mu_{t}\right) S\right]_{L} .
$$


2.4. One-Equation SGS Model. A disadvantage of the Smagorinsky model is the loss of information resulting from use of the deviatoric part of the SGS stress tensor only. In CFX-4, we have implemented a transport equation for SGS kinetic energy $\left(K_{\mathrm{SGS}}\right)$, defined by

$$
k_{\mathrm{SGS}, L}=\frac{1}{2 \rho_{L}} \tau_{k k, L},
$$

of the following transport form [12]:

$$
\begin{aligned}
& \frac{\partial\left(r_{L} \rho_{L} k_{\mathrm{SGS}}\right)}{\partial t}+\nabla \cdot\left(r_{L} \rho_{L} \mathbf{u}_{L} k_{\mathrm{SGS}}\right) \\
& \quad=\nabla\left[r_{L}\left(\mu_{L}+\mu_{t, L}\right) \nabla k_{\mathrm{SGS}}\right]=+r_{L} P_{k_{\mathrm{SGS}}}-r_{L} \rho_{L} C_{\varepsilon} \frac{k_{\mathrm{SGS}}^{3 / 2}}{\Delta},
\end{aligned}
$$

where $P_{k_{\mathrm{SGS}}}$ is the turbulence production term defined as:

$$
P_{k_{\mathrm{SGS}, L}}=\left(\mu_{t} \mathbf{S}: \mathbf{S}\right)_{L}
$$

Using this model, the eddy viscosity is then calculated from:

$$
\mu_{t, L}=C_{k} \rho_{L} \Delta k_{\mathrm{SGS}, L}^{1 / 2},
$$

with model constants $C_{k}=0.07$ and $C_{\varepsilon}=1.05$. A detailed description of the one-equation SGS model for dispersed bubbly flows can be found in [12].

2.5. Interfacial Forces. To close the momentum equation set for the two phases, the various interphase exchange terms have to be modelled. The interphase exchange terms relevant for the configuration considered in this work are drag, buoyancy, virtual mass, and lift forces. In the framework of the EELES presented in this work, interfacial forces are applied only to the resolved field, while their SGS counterparts are neglected.

The drag force is modelled from the resolved velocity field as follows [13]:

$$
\mathbf{M}_{D, L}=-\mathbf{M}_{D, G}=\frac{3}{4} r_{G} \rho_{L} \frac{C_{D}}{d_{G}}\left|\mathbf{u}_{G}-\mathbf{u}_{L}\right|\left(\mathbf{u}_{G}-\mathbf{u}_{L}\right),
$$

where $C_{D}$ is the drag coefficient, defined here in terms of the Eötvös number $\left(E_{O}=g \Delta \rho d_{G}^{2} / \sigma\right)$ :

$$
C_{D}=\frac{2}{3} E_{0}^{1 / 2}
$$

In the present application, a bubble size of $4.0 \mathrm{~mm}$ is specified (the value reported in [5]), and the coefficient of surface tension $\sigma=0.072 \mathrm{~N} / \mathrm{m}$, giving $E_{O}=2.2$ and $C_{D}=$ 1.0 .

For the virtual mass force, we use the model of Drew and Lahey Jr. [14]:

$$
\mathbf{M}_{\mathrm{VM}, L}=-\mathbf{M}_{\mathrm{VM}, G}=r_{G} \rho_{L} C_{\mathrm{VM}}\left(\frac{D \mathbf{u}_{G}}{D t}-\frac{D \mathbf{u}_{L}}{D t}\right),
$$

where $C_{V M}$ is the virtual mass force coefficient, here set to 0.5 .

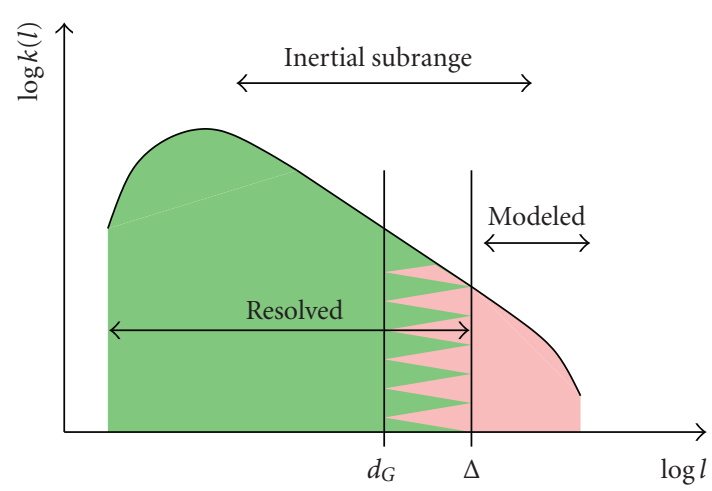

Figure 2: Bubble size larger than filter width.

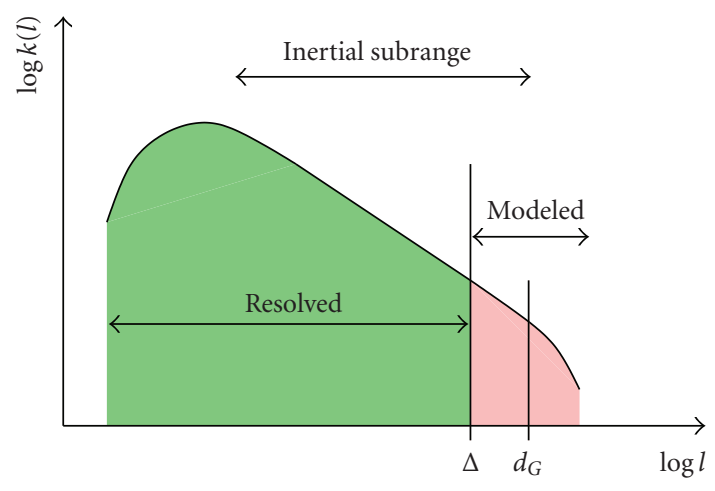

Figure 3: Bubble size smaller than filter width.

The lift force accounts for the transverse migration of bubbles under the influence of the liquid shear field. Following earlier work $[14,15]$, it is modelled here according to

$$
\mathbf{M}_{L, L}=-\mathbf{M}_{L, G}=\rho_{L} C_{L}\left(\mathbf{u}_{G}-\mathbf{u}_{L}\right) \times \nabla \times \mathbf{u}_{L},
$$

in which the lift coefficient, $C_{L}$, is set to 0.5 .

\section{Requirements for the Numerical Grid}

In the present work, we couple the Euler-Euler approach for multiphase flow with LES, and therefore have to consider the resolution requirements of both techniques simultaneously in order to choose a satisfactory grid. A basic requirement is that the control volume size should be large enough to encompass all the interface details. This is the intrinsic assumption in the derivation of the Euler-Euler model equations, and strictly has to be satisfied at the discrete level as well.

In LES, the SGS model is often very simple, and only drains energy from the resolved field without feed-back. Therefore, our goal in LES is to resolve as much of the flow field as possible, and to have as fine a grid as feasible for the available computer hardware. Since the Euler-Euler approach specifies the minimum control volume size, whereas for LES we are invariantly seeking as fine a grid as possible, the requirements for the numerical grid may sometimes 


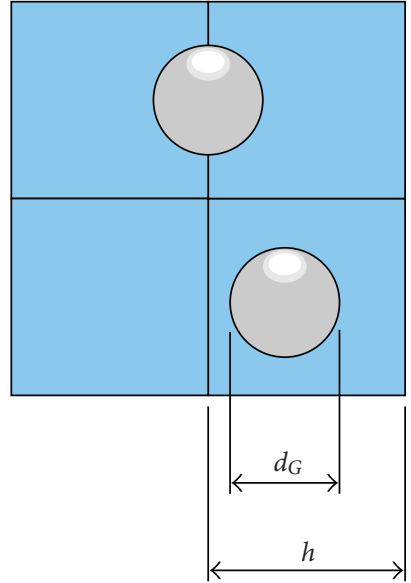

Figure 4: The Milelli condition.

be in conflict. The point is illustrated in Figures 2 and 3, which show the turbulence spectra under different modelling conditions. For a successful LES, we must have a filter width $(\Delta)$ in the inertial subrange region, and all scales of motion larger than that (left of $\Delta$ in Figures 2 and 3), must be accurately resolved on the numerical grid. If, however, we have a bubble diameter $\left(d_{G}\right)$ larger than $\Delta$ (see Figure 2), it is obvious that they would induce some large-scale motions which are not properly accounted for by the EELES, since there is no information on the interface details or their influence on the resolved large-scale motions. If in addition we use a model for bubble-induced turbulence, it would drain the energy from the resolved field, further deteriorating the accuracy of the resolved field. This is illustrated by the saw-like shading in Figure 2. This influence on the resolved part of the spectra is not acceptable for LES. This is not just a conceptual consideration, as discussed in [6], and it is explained in more detail in Section 3.1. The situation which is safe for EELES is shown in Figure 3 , where $d_{G}$ is smaller than $\Delta$, and all bubble-induced scales which cannot be calculated using EELES approach fall into the SGS part of the spectra.

3.1. The Milelli Condition. The grid size considerations discussed above, are not new. In the present work, we have indicated why the bubbles must be smaller than the cell size from the point of view of the energy spectra and modelling closures for the interfacial forces. A systematic a posteriori analysis of the minimum ratio of the bubble and cell sizes for LES modelling of free bubble plumes is reported by Milelli et al. [6], which resulted in the following criterion:

$$
\frac{h}{d_{G}} \geq 1.5 \text {. }
$$

Equation (18) states that the cell size must be at least $50 \%$ larger than the bubble diameter for accurate LES. The situation is illustrated in Figure 4. In the Deen experiment, the mean bubble size is $4 \mathrm{~mm}$. Since the flow is dominated by the energetic, large-scale structures in the core of the flow, with wall effects having a smaller impact on the overall flow field, we have created a uniform grid with $30 \times 30 \times 100$ cubic cells. This results in a cell size of $5 \mathrm{~mm}$, hence,

$$
\frac{h}{d_{G}}=1.2
$$

which is below the optimum value. A coarser mesh which satisfies the Milelli condition has also been used, but with no significant change in the computed results [12].

It might be argued at this point that the grid used in the present simulations is too coarse for LES, at least from the point of view of capturing all the relevant (large) scales. Judging from the flow patterns that can be expected in a square column such as this, with the bubble plume meandering from one direction to the other, it is quite conceivable that the largest, and therefore the most energetic, eddies will be of the size of the domain cross-section. Therefore, we are confident that the grid resolution we employ $(30 \times 30$ cells in the cross-section) is sufficient to resolve these large structures. Should the topology of the flow be different, and should the flow be dominated by small, near-wall scales of motion, this resolution would not be adequate. The adequacy of the grid resolution used is proved in the following section.

\section{Simulation Details}

In this section, we briefly summarize all the relevant simulation details.

Inlet Superficial Velocities:

for liquid: $J_{L}=0.0 \mathrm{~m} / \mathrm{s}$,

for gas: $J_{G}=0.0049 \mathrm{~m} / \mathrm{s}$.

Spatial Discretization:

number of cells in computational domain: 90 000;

advection scheme: bounded central scheme for Neptune_CFD;

quick scheme for CFX-4.

\section{Temporal Discretization:}

the linear backward time differencing for Neptune_ CFD;

quadratic backward differencing for CFX-4;

$\mathrm{CFL}=1.0$, variable time step, approximately equal to 0.05 second for Neptune_CFD constant time step of 0.05 second for CFX-4, leading to $\mathrm{CFL} \approx 1.0$;

integral time of simulation: 400 seconds;

simple time-average is used in both, not weighted by volume fraction. 


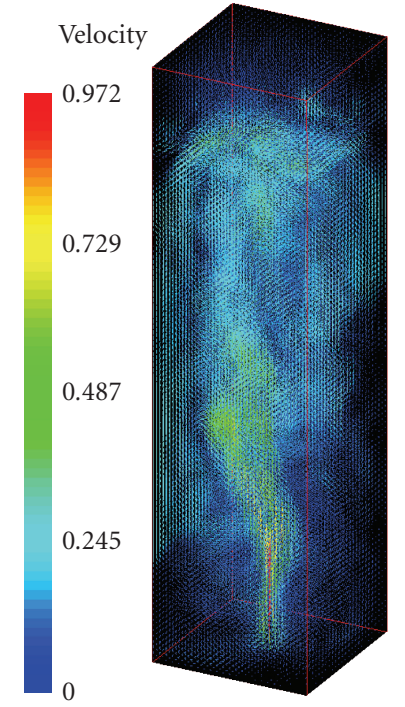

(a)

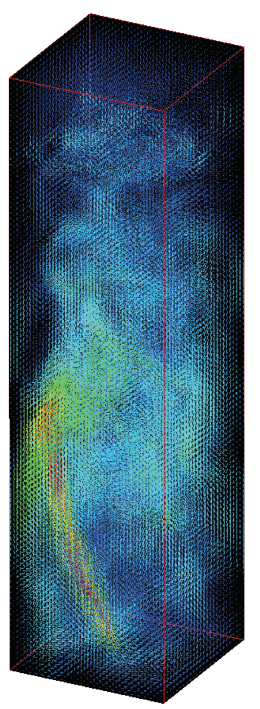

(b)

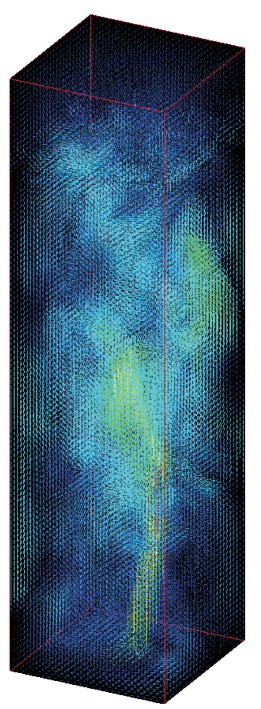

(c)

Figure 5: Instantaneous velocity vectors, calculated using Neptune_CFD, at instants: 60 seconds, 80 seconds, and 120 seconds.

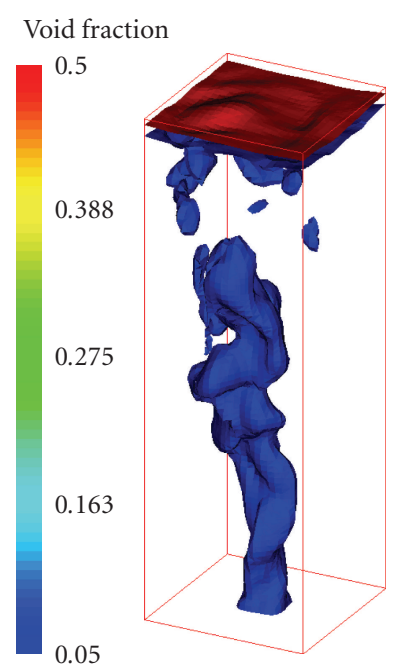

(a)

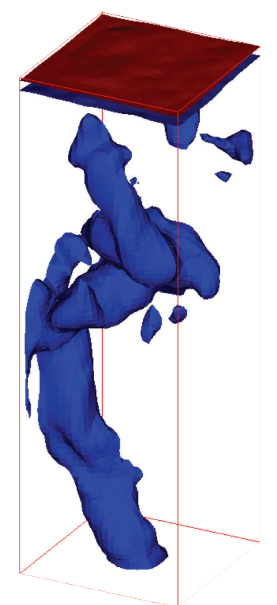

(b)

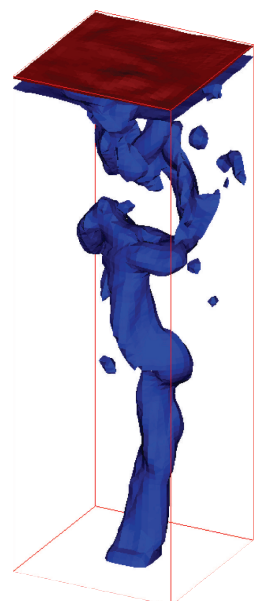

(c)

Figure 6: Isosurfaces of constant void fraction (0.05 and 0.5), calculated using Neptune_CFD, at instants: 60 seconds, 80 seconds, and 120 seconds.

\section{Results}

In this section, the results for our EELES with Neptune_CFD are presented. We start with the instantaneous velocity and void fraction fields to show the flow patterns in the bubble column flow, and continue with time-averaged velocity and fluctuating liquid velocity profiles. Time-averaged profiles obtained with Neptune_CFD and CFX-4 are compared with the experimental measurements of Deen et al. [5].

5.1. Instantaneous Results. Instantaneous velocity fields are shown in Figure 5. Velocity vectors are coloured with the velocity magnitude. Two things are immediately apparent. First, the velocity field does not show any signs of the growing instabilities to which LES computations are very often prone. This is not surprising, since the advection scheme is bounded. Another thing worth noting is that the velocity field pattern is changing dramatically in time. Bubbles are moving up through the large turbulent structures, driven by their buoyancy, resulting in different bubble paths at each time instant. This behaviour was also reported for bubble plume experiments [16]. Figure 6 shows two isosurfaces of constant void fraction. The blue one corresponds to the lowvoid fraction of $r=.05$ and the red to $r=.5$. The threshold for the blue isosurface gives a good illustration of the bubble plume shape, whereas the red one indicates the position of the free surface. 


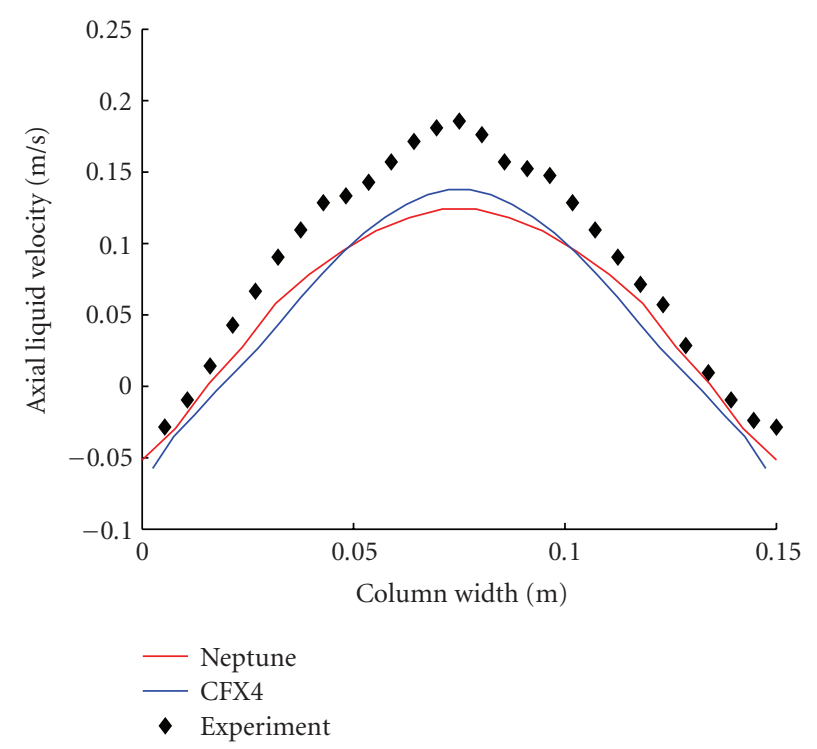

(a)

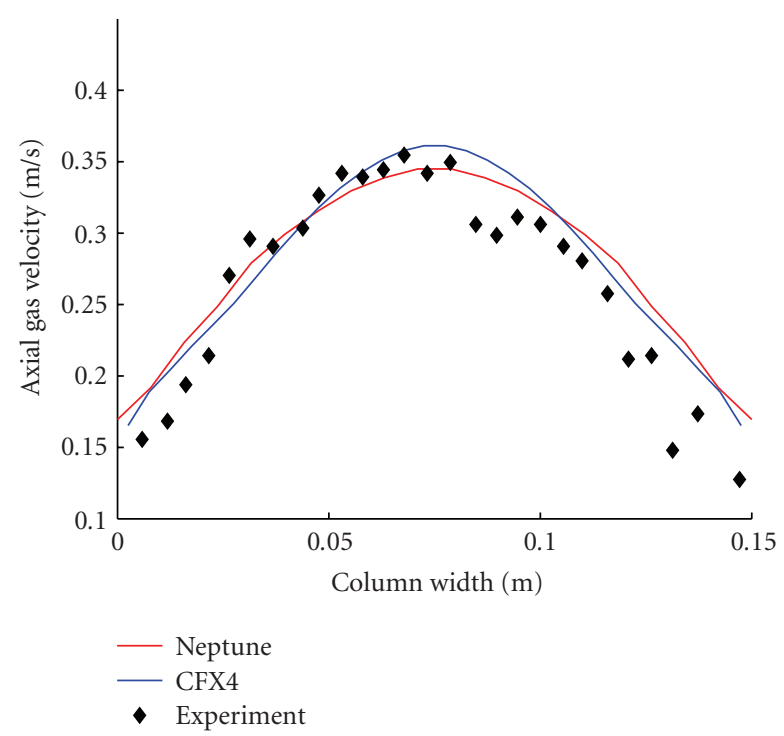

(b)

Figure 7: Comparison of (a) liquid and (b) gas velocity profiles obtained with CFX-4 using a one-equation model, Neptune_CFD with the Smagorinsky model, and experimental data.

5.2. Time-Averaged Results. In this section, we compare the simulated profiles of liquid and gas vertical velocities and liquid turbulence intensities against the reported experimental data [5]. The measurements were taken along the centreline of the horizontal plane at height $25 \mathrm{~cm}$.

Figure 7 shows comparisons of vertical liquid and gas vertical velocity profiles. The liquid velocity profile is somewhat under-predicted by both codes. In contrast, the gas velocity prediction compares very well with experiment in both cases. From these time-averaged results, it might be concluded that the drag force is underestimated in both codes, probably due to a wrong assumption for the drag

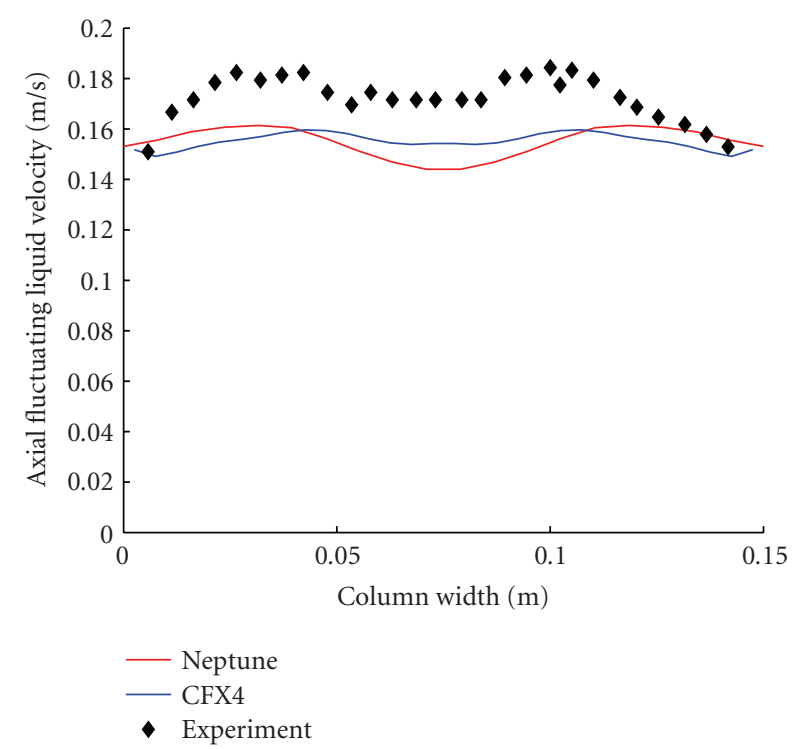

(a)

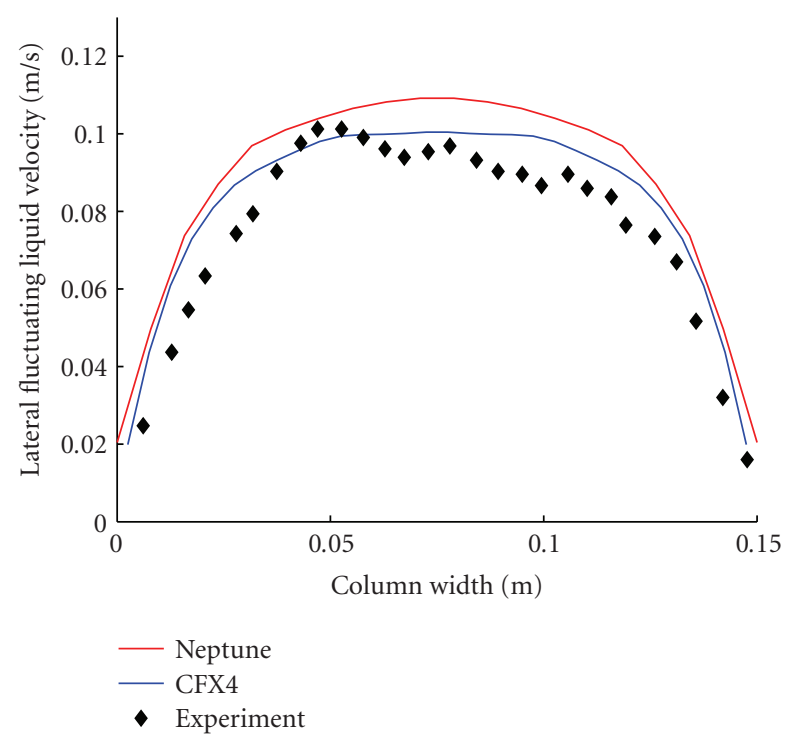

(b)

Figure 8: Comparison of (a) vertical and (b) lateral fluctuating liquid velocities, obtained using CFX-4 with a one-equation model, Neptune_CFD with the Smagorinsky model, and experimental data.

coefficient $\left(C_{D}\right)$, or for the relative velocity between the two phases [16].

The vertical component of the resolved vertical fluctuating liquid velocity is plotted in Figure 8(a). The qualitative comparison is encouraging for both codes, since the twinpeaked shape seen in the experiments is reproduced. Quantitatively, however, both codes under-predict the magnitude to some extent. Figure 8(b) shows the resolved lateral fluctuating liquid velocity, which is predicted very well. The total turbulent kinetic energy, plotted in Figure 9, in spite of the under-prediction of vertical fluctuating liquid component, is well predicted by both codes in the middle 


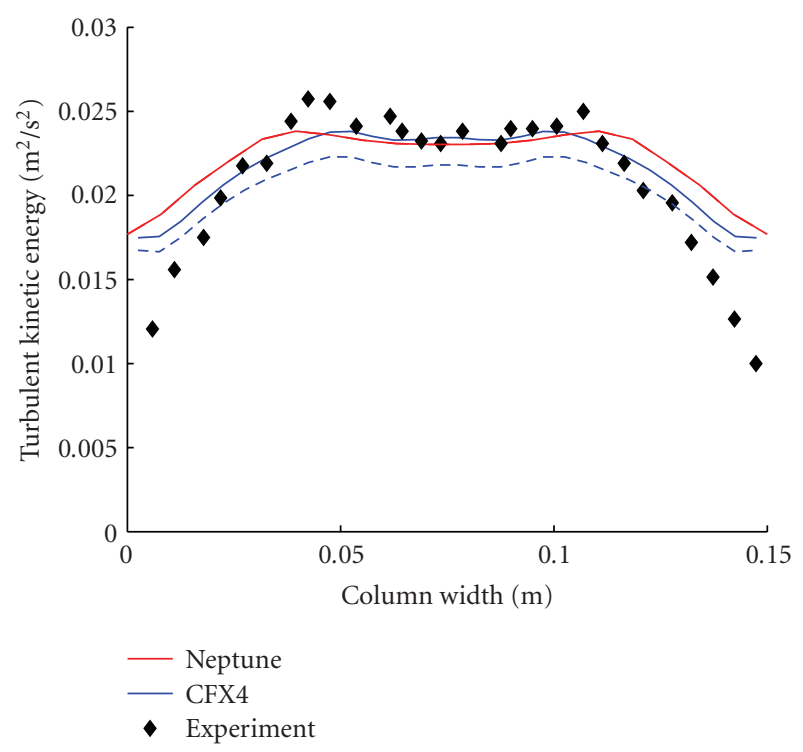

Figure 9: Comparison of liquid turbulent kinetic energy obtained with CFX-4 using a one-equation model, Neptune_CFD with the Smagorinsky model, and experimental data. The blue dashed line is the resolved, the blue continuous line is the total (resolved plus SGS) kinetic energy.

of the column and somewhat over-predicted close to the walls. This over-estimation of the near-wall kinetic energy might be attributed to insufficient resolution for the nearwall structures.

\section{Conclusions and Perspectives}

Implementation of the Smagorinsky SGS model in the Neptune_CFD code has been validated using data from the Deen bubble column experiment. Results have also been compared with predictions obtained from simulations performed using the code CFX-4. Generally, liquid velocity profiles are under-predicted compared with measured data, whereas the gas velocity profiles are predicted very well. This can only be attributed to a wrong assumption for the drag coefficient. The liquid velocity fluctuations predicted by both codes compare well with experiments, except for some under-prediction of the vertical component. Overall, the results obtained with the two codes are consistent, regardless of the fact that different SGS models have been used. This is a clear indication that the SGS model influences results only to a very small extent.

When envisaging the potential of LES for modelling multiphase flows in general, one should clearly distinguish between the scales at which LES might be used, since this implies the level of detail of accurate interface resolution to the degree of modelling that can be tolerated. Simulations at mesoscales imply an Euler-Euler description of the interface between the phases, such as the one described in this work. However, if LES for multiphase flows is applied at microscales, explicit interface tracking procedures would be needed. This has already been proposed in [17], and is generally referred to as large-scale simulation (LSS).

The principal advantage of EELES over LSS is that since the interface details are not calculated explicitly, the simulations may be carried out at lower cost. The principal disadvantage of EELES is that the most influential interfacial forces (lift and drag) are modelled for the large-scale field, meaning that the question of how to model these forces remains as open for EELES as it is with RANS. LSS, on the other hand, explicitly resolves the large-scale part of the interfacial forces, leaving the modelling at the SGS level, where the effects are smaller and hence less influential on the accuracy of the results. LSS modelling comes at a heavy price, due to the need to resolve all relevant interface details, imposing huge demands on computing power. Applying LSS to industrial-scale problems is beyond the current state of computing resources.

Another disadvantage of EELES stems from the different resolution requirements imposed by the Euler-Euler description of the two fluids and that of the LES approach itself, expressed in [6]. Since for an Euler-Euler description, the minimum cell size must be larger than the interface detail, accurate LES requires as fine a grid as possible. This requirement is particularly stringent in the near-wall regions, where the turbulent structures (e.g., streaks) are very small, and a very fine grid is needed to capture all relevant details. The disadvantages of EELES just outlined are not so pronounced for flows in which the bubbles are small compared to the grid size, such as for the bubble column studied in this work. Generally, one should use EELES if the flow is likely to be unsteady, with large coherent structures generated either by buoyancy or obstacles in the flow. EELES, as LES itself, should be avoided for wall-dominated flows. In other words, large eddy simulation should be used in the cases in which large eddies are present. Flows with density stratification in large channels (relevant to the PTS issue), in addition to bubble column devices, are examples where EELES has the potential to give accurate insight into the phenomena taking place and the ability to quantify them.

\section{References}

[1] D. Cacuci, "European Platform for Nuclear Reactor simulation (NURESIM)," Integrated project NUCTECH-20043.4.3.1-1. EURATOM Research and Training Programme on Nuclear Energy, 2004.

[2] D. Bestion, H. Anglart, P. Peturaud, et al., "Review of available data for validation of NURESIM two-phase CFD software applied to CHF investigations," in Proceedings of the 12th International Topical Meeting on Nuclear Reactor Thermal Hydraulics (NURETH '07), Pittsburgh, Pa, USA, SeptemberOctober 2007.

[3] D. Lucas, D. Bestion, E. Bodèle, et al., "On the simulation of two-phase flow pressurized thermal shock (PTS)," in Proceedings of the 12th International Topical Meeting on Nuclear Reactor Thermal Hydraulics (NURETH'07), Pittsburgh, Pa, USA, September-October 2007.

[4] N. Méchitoua, M. Boucker, J. Laviéville, J. Hérard, S. Pigny, and G. Serre, "An unstructured finite volume solver for 
two-phase water/vapour flows modeling based on an elliptic oriented fractional step method," in Proceedings of the 10th International Topical Meeting on Nuclear Reactor ThermalHydraulics (NURETH '03), Seoul, Korea, October 2003.

[5] N. G. Deen, T. Solberg, and B. H. Hjertager, "Large eddy simulation of the gas-liquid flow in a square cross-sectioned bubble column," Chemical Engineering Science, vol. 56, no. 2122, pp. 6341-6349, 2001.

[6] M. Milelli, B. L. Smith, and D. Lakehal, "Large-eddy simulation of turbulent shear flows laden with bubbles," in Direct and Large-Eddy Simulation-IV, B. J. Geurts, R. Friedrich, and O. Metais, Eds., vol. 8 of ERCOFTAC Series, pp. 461-470, Kluwer Academic Publishers, Dordrecht, The Netherlands, 2001.

[7] N. G. Deen, B. H. Hjertager, and T. Solberg, "Comparison of PIV and LDA measurement methods applied to the gasliquid flow in a bubble column," in Proceedings of the 10th International Symposium on Applications of Laser Techniques to Fluid Mechanics, Lisbon, Portugal, July 2000.

[8] D. Lakehal, B. L. Smith, and M. Milelli, "Large-eddy simulation of bubbly turbulent shear flows," Journal of Turbulence, vol. 3, no. 25, pp. 1-21, 2002.

[9] B. N. Vanga Reddy, M. A. Lopez de Bertodano, E. Krepper, A. Zaruba, and H. M. Prasser, "Two-fluid model LES of a bubble column," in Proceedings of the 11th International Topical Meeting on Nuclear Reactor Thermal-Hydraulics (NURETH'05), Avignon, France, October 2005.

[10] J. Smagorinsky, "General circulation experiments with the primitive equations," Monthly Weather Review, vol. 91, no. 3, pp. 99-165, 1963.

[11] J. B. Joshi, V. S. Vitankar, A. A. Kulkarni, M. T. Dhotre, and K. Ekambara, "Coherent flow structures in bubble column reactors," Chemical Engineering Science, vol. 57, no. 16, pp. 3157-3183, 2002.

[12] B. Ničeno, M. T. Dhotre, and N. G. Deen, "One-equation subgrid scale (SGS) modeling of Euler-Euler large eddy simulation (EELES) of dispersed bubbly flow," to appear in Chemical Engineering Science.

[13] M. Ishii and N. Zuber, "Drag coefficient and relative velocity in bubbly, droplet or particulate flows," AIChE Journal, vol. 25, no. 5 , p. $843,1979$.

[14] D. A. Drew and R. T. Lahey Jr., "Virtual mass and lift force on a sphere in rotating and straining inviscid flow," International Journal of Multiphase Flow, vol. 13, no. 1, pp. 113-121, 1987.

[15] I. Žun, "The mechanism of bubble non-homogeneous distribution in two-phase shear flow," Nuclear Engineering and Design, vol. 118, pp. 155-162.

[16] M. Simiano, "Experimental investigation of large-scale three dimensional bubble plume dynamics," Dissertation, Swiss Federal Institute of Technology, Zurich, Switzerland, 2005.

[17] A. Alajbegovic, "Large eddy simulation formalism applied to multiphase flows," in Proceedings of the ASME Fluids Engineering Division Summer Meeting (FEDSM '01), pp. 529534, New Orleans, La, USA, May-June 2001. 

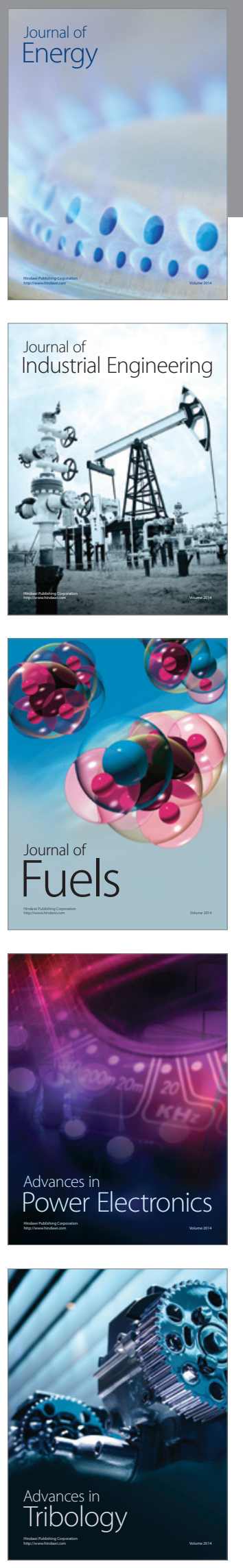
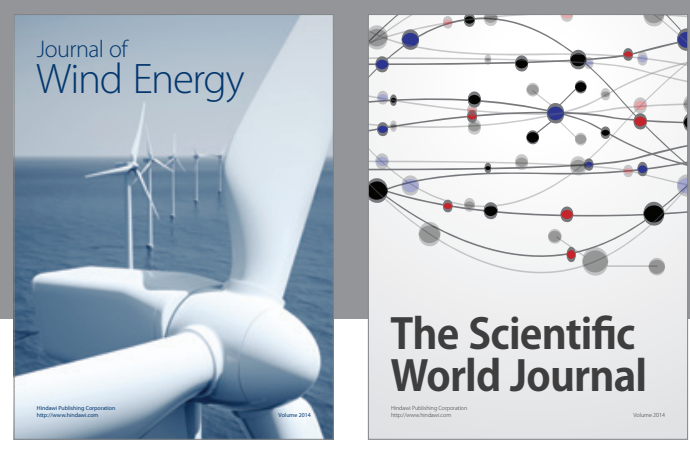

The Scientific World Journal

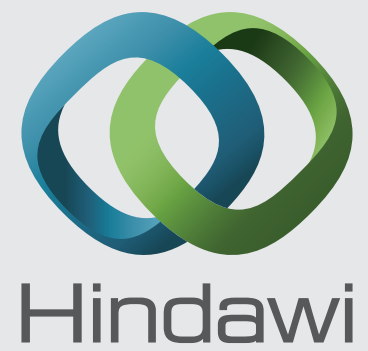

Submit your manuscripts at http://www.hindawi.com
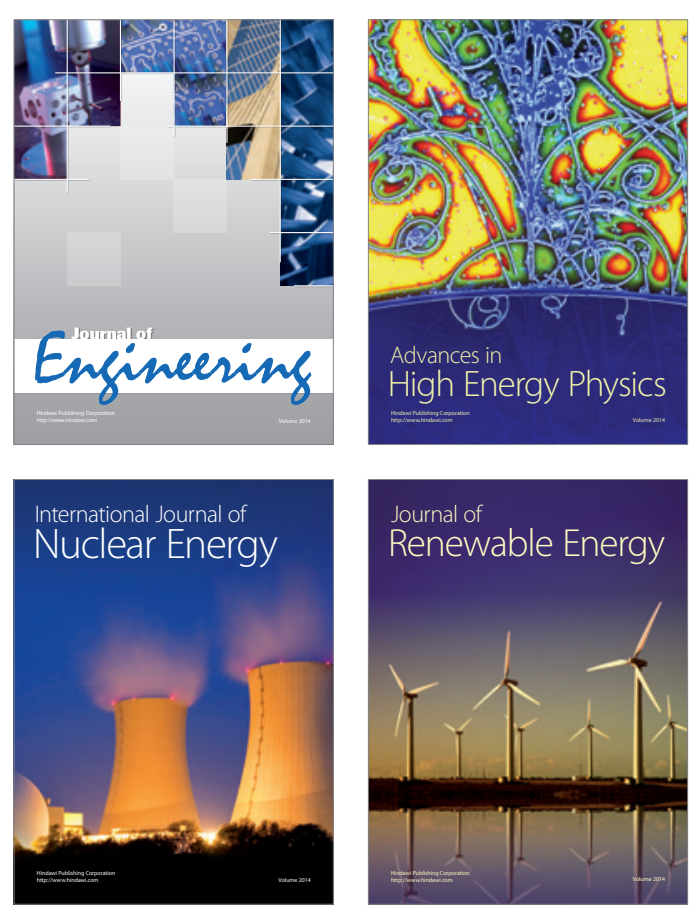

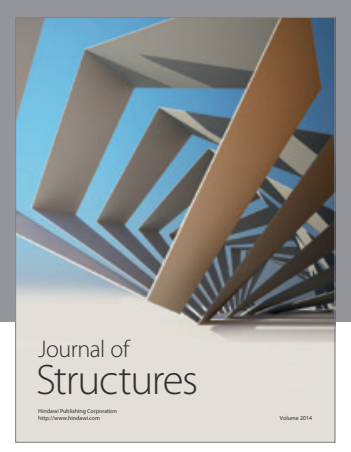

Rotating
Mechinery
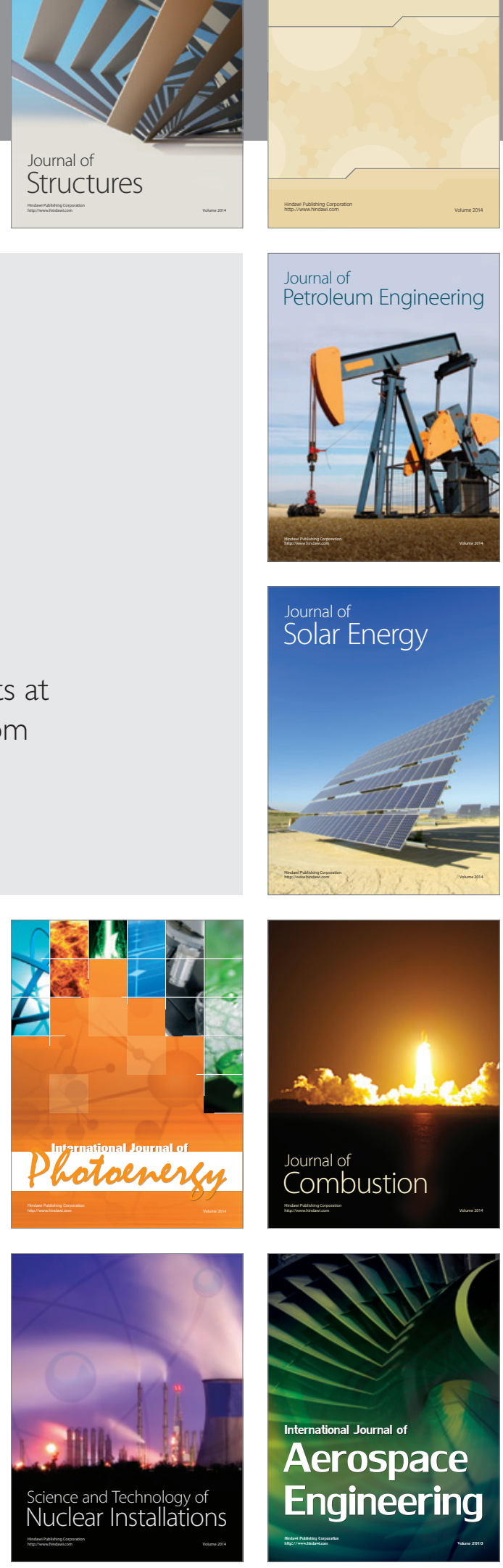